\title{
Quantum buoyancy, generalized second law, and higher-dimensional entropy bounds
}

\author{
Shahar Hod \\ The Ruppin Academic Center, Emeq Hefer 40250, Israel \\ and
}

The Hadassah Institute, Jerusalem 91010, Israel

(Dated: August 28, 2018)

\begin{abstract}
Bekenstein has presented evidence for the existence of a universal upper bound of magnitude $2 \pi R / \hbar c$ to the entropy-to-energy ratio $S / E$ of an arbitrary three dimensional system of proper radius $R$ and negligible self-gravity. In this paper we derive a generalized upper bound on the entropy-toenergy ratio of a $(D+1)$-dimensional system. We consider a box full of entropy lowered towards and then dropped into a $(D+1)$-dimensional black hole in equilibrium with thermal radiation. In the canonical case of three spatial dimensions, it was previously established that due to quantum buoyancy effects the box floats at some neutral point very close to the horizon. We find here that the significance of quantum buoyancy increases dramatically with the number $D$ of spatial dimensions. In particular, we find that the neutral (floating) point of the box lies near the horizon only if its length $b$ is large enough such that $b / b_{C}>F(D)$, where $b_{C}$ is the Compton length of the body and $F(D) \sim D^{D / 2} \gg 1$ for $D \gg 1$. A consequence is that quantum buoyancy severely restricts our ability to deduce the universal entropy bound from the generalized second law of thermodynamics in higher-dimensional spacetimes with $D \gg 1$. Nevertheless, we find that the universal entropy bound is always a sufficient condition for operation of the generalized second law in this type of gedanken experiments.
\end{abstract}

\section{INTRODUCTION}

One of the most intriguing features of both the classical and quantum theory of black holes is the striking analogy between the laws of black-hole physics and the universal laws of thermodynamics [1-3]. In particular, Hawking's (classical) theorem [2], "The surface area of a black hole never decreases", is a property reminiscent of the entropy of a closed system. This remarkable analogy had led Bekenstein [4] to conjecture that the area of a black hole (in suitable units) may be regarded as the black-hole entropy - entropy in the sense of inaccessible information about the internal state of the black hole. This conjecture is logically related to a second conjecture, known as the generalized second law of thermodynamics (GSL) [4]: "The sum of the black-hole entropy (1/4 of the horizon's surface area) and the common (ordinary) entropy in the black-hole exterior never decreases."

Arguing from the GSL, Bekenstein [5] has proposed the existence of a universal upper bound on the entropy $S$ (and hence information capacity) of any weakly selfgravitating physical system of circumscribing radius $R$ and total energy $E$ :

$$
S \leq \frac{2 \pi R E}{\hbar c}
$$

This bound was deduced from the requirement that the GSL be respected when a box containing entropy is placed with no radial motion near the horizon of a black hole, and then allowed to fall in. The entropy of the box disappears but an increase in the black hole entropy occurs. The GSL is respected provided the box's entropy $S$ is bounded as in (11).

It is worth mentioning that, the canonical Bekenstein bound (11) can be tightened: an improved upper bound to the entropy of a spinning object was obtained in $[6]$ and an improved bound to the entropy of a charged system was found in 7, 8]. For a cubic system, a somewhat stronger bound can be deduced from the GSL with $2 R$ replaced by the cubic length $b$ :

$$
S \leq \frac{\pi b E}{\hbar c} .
$$

The main purpose of the present paper is to extend Bekenstein's analysis to higher dimensional spacetimes. We shall find below that in the generic case of $D$ flat spatial dimensions, the bound (2) may be deduced from the GSL only under severe restrictions on the size of the system.

\section{ACCELERATION RADIATION}

In the original derivation [5] of the universal entropy bound it was assumed that the energy (as measured at infinity) added to the black hole with the box is that which may be inferred from the red-shift factor at the deposition point (this energy determines the increase in black-hole entropy). However, it was pointed out in [9] that when the deposition of the box is attempted by lowering it from far away, buoyancy in the radiative black hole environs will prevent lowering the box all the way down to the horizon [10]. In fact, it was realized [9] that one must invest energy in order to push the box into the black hole against the quantum buoyant force [10]. This extra energy contributes to the black-hole area increase, helping to ensure the validity of the GSL in this gedanken experiment.

An observer accelerating with acceleration $a$ detects 
isotropic thermal radiation with temperature

$$
T_{U}=\frac{\hbar a}{2 \pi k_{B} c},
$$

the celebrated Unruh radiance [9]. An object hanging above a black hole is accelerated by virtue of its being prevented from following a geodesic [11]. It was suggested [9] that this object will likewise see Unruh radiance. Because its acceleration (and hence the temperature $T_{U}$ ) varies with the height above the horizon, it was concluded that the object will be influenced by a quantum buoyant force. This buoyant force is due to the non-uniformity of the ambient pressure (non-uniformity of the Unruh temperature) [11].

Two intriguing consequences were inferred [9 11]:

- The buoyancy can cause an object sufficiently near the horizon to float at some neutral point.

- The buoyancy contributes to the energy bookkeeping of a process in which a box is lowered from far away towards a black hole while doing work on the lowering machine. In particular, the total energy at infinity added to the hole after the box has been dropped from the neutral point is larger than the red-shifted proper energy of the box.

It should be emphasized that it was shown in [10] that for generic systems in three spatial dimensions the neutral point lies very near the horizon. A consequence is that in three spatial dimensions, the buoyancy makes only a negligible contribution to the energy bookkeeping of the gedanken experiment. This implies that the original entropy bounds (10)-(2) can be deduced if the GSL is assumed to hold. However, in this paper we shall show that this conclusion may not be valid in generic $(D+1)$ dimensional spacetimes with $D \gg 1$.

We shall carry out the gedanken experiment with a $(D+1)$-dimensional Schwarzschild-Tangherlini black hole [12, 13] of ADM mass $\mathcal{M}$. The exterior spacetime is described by the metric (we use natural units in which $G=c=k_{B}=1$ )

$$
d s^{2}=-H(r) d t^{2}+H(r)^{-1} d r^{2}+r^{2} d \Omega^{(D-1)},
$$

where

$$
H(r)=1-\left(\frac{r_{H}}{r}\right)^{D-2} \equiv[\chi(r)]^{2}
$$

defines the redshift factor, $\chi(r)$. Here

$$
r_{H}=\left[\frac{16 \pi \mathcal{M}}{(D-1) A_{D-1}}\right]^{\frac{1}{D-2}}
$$

is the black hole's radius and

$$
A_{D-1}=\frac{2 \pi^{D / 2}}{\Gamma(D / 2)}
$$

is the area of a unit $(D-1)$-sphere.
Following [9 11] we assume the black hole has reached equilibrium with its own Hawking radiation, the whole system being enclosed in a large cavity. The black hole temperature $T_{b h}$ and the local temperature $T$ are related by 9011

$$
T=\frac{T_{b h}}{\chi}=\frac{(D-2) \hbar}{4 \pi r_{H} \chi} .
$$

In the near-horizon region, $r-r_{H} \ll r_{H}$, the redshift factor is given by

$$
\chi^{2}(r)=(D-2) \frac{r-r_{H}}{r_{H}}\left[1+O\left(\left(r-r_{H}\right) / r_{H}\right)\right] .
$$

The radial coordinate $r$ is related to the proper distance $l$ above the horizon through the relation

$$
\begin{aligned}
& l(r)=\int_{r_{H}}^{r}\left[\chi\left(r^{\prime}\right)\right]^{-1} d r^{\prime} \\
& =2\left[\frac{r_{H}\left(r-r_{H}\right)}{D-2}\right]^{1 / 2}\left[1+O\left(\left(r-r_{H}\right) / r_{H}\right)\right] .
\end{aligned}
$$

From (9) and (10) one obtains the near-horizon relation

$$
\chi(l)=\frac{D-2}{2 r_{H}} l .
$$

Taking cognizance of Eq. (8), one can write the local temperature as

$$
T=\frac{\hbar}{2 \pi l} .
$$

Note that one could also obtain (12) from the observation [10, 11] that a point suspended at rest in a $(D+1)$ dimensional Schwarzschild spacetime is characterized by an invariant acceleration $a=d \chi / d r$. Taking cognizance of Eqs. (3) and (11), one obtains the formal Unruh temperature $\hbar / 2 \pi l$.

\section{ENERGETICS OF THE SYSTEM}

The main goal of this paper is to extend Bekenstein's analysis to higher dimensional spacetimes, properly taking into account the contribution of quantum buoyancy to the energy bookkeeping of the lowering process. Ignoring the effects of quantum buoyancy, it was found in 14] that the bound (1) can be inferred from the GSL in $(D+1)$-dimensional spacetimes for any value of $D$. However, we shall find below that the contribution of quantum buoyancy to the energy bookkeeping increases dramatically with the number of spatial dimensions $D$. As a consequence, we shall show that the argument justifying the bound (1) from the GSL cannot be completed for $D \gg 1$ unless the size of the object is very large [see Eqs. (34)- (35) below].

The system we consider is a $(D+1)$-dimensional hypercube with sides of length $b$, rest mass $\mu$, and which 
holds entropy $S$. To obtain an entropy bound, one may lower the system from infinity into a $(D+1)$-dimensional black hole. The test-particle approximation imposes the constraint $r_{H}(\mu) \ll b \ll r_{H}(\mathcal{M})$. This guarantees that the object has a negligible self-gravity and that it is much smaller than the size of the black hole. One would like to add as little energy as possible to the black hole. This will minimize the corresponding increase of the black hole's surface area and thus will help to optimize the tightness of the entropy bound [5, 14]. Therefore, the strategy is to extract work from the system by lowering the box slowly towards the black hole, before one finally drops it in.

The total energy $\mathcal{E}$ (energy-at-infinity) of a body located at a radial coordinate $r$ in the black-hole spacetime is made up of two contributions [10, 11]:

- $\mathcal{E}_{0}=\mu \chi(r)$, the energy associated with the body's mass (red-shifted by the gravitational field). Taking cognizance of Eq. (11), one finds

$$
\mathcal{E}_{0}=\frac{D-2}{2 r_{H}} \mu l
$$

- The contribution to the energy from the work done to overcome the buoyancy is 10,11 ]

$$
W_{\text {buoy }}=\int_{l}^{\infty} f_{\text {buoy }} d l^{\prime}
$$

where $l$ is the proper height of the centroid plane of the box above the horizon, and $f_{\text {buoy }}$ is the buoyant force acting on the box, as measured by an observer at infinity.

The buoyant force acting on the box, as measured by an observer at infinity, is the difference of the redshifted local forces acting on the upper and lower faces [9 11]:

$$
f_{\text {buoy }}(l)=A\left[(P \chi)_{l-b / 2}-(P \chi)_{l+b / 2}\right]
$$

where $A=b^{D-1}$ is the horizontal crossectional area of the box and $P$ is the radiation pressure. As a consequence of the cancelation of the work done by buoyant forces on top and bottom of the box over the range $[l+b / 2, \infty]$, the buoyant contribution to the energy $\mathcal{E}$ of the body only depends on the distribution of $P \chi$ over the height of the box. Taking cognizance of (15) one therefore finds [9 11$]$

$$
W_{\text {buoy }}=A \int_{l-b / 2}^{l+b / 2} P \chi d l^{\prime} .
$$

Following [9 11] we shall assume a model of acceleration radiation as a mixture of noninteracting thermal gases of massless particles. The mean energy density of thermal radiation in $D$ spatial dimensions for one helicity degree of freedom is given by [10]

$$
e=\int_{0}^{\infty} \frac{\hbar \omega d V_{D}(\omega)}{\left(e^{\hbar \omega / T} \mp 1\right)(2 \pi)^{D}}
$$

where the upper (lower) signs correspond to boson (fermion) fields, and

$$
d V_{D}(\omega)=\left[2 \pi^{D / 2} / \Gamma(D / 2)\right] \omega^{D-1} d \omega
$$

is the volume in frequency space of the shell $(\omega, \omega+d \omega)$.

From Eqs. (17)-(18) and the relation

$\int_{0}^{\infty} \frac{x^{D} d x}{e^{x} \mp 1}=\zeta(D+1) \Gamma(D+1) \times \begin{cases}1 & \text { for bosons } \\ 1-2^{-D} & \text { for fermions }\end{cases}$

where $\zeta(z)$ is the Riemann zeta function, one finds that the mean energy density of all massless fields is given by

$$
e=\frac{N D \zeta(D+1) \Gamma\left(\frac{D+1}{2}\right) T^{D+1}}{\pi^{\frac{D+1}{2}} \hbar^{D}},
$$

where $N$ is the effective number of massless degrees of freedom (the number of polarization states). Massless scalars contribute 1 to $N$, massless fermions contribute $1-2^{-D}$ to $N$ [10], an electromagnetic field contributes $D-1$ to $N$ [15], and the graviton contributes $(D+1)(D-$ $2) / 2$ to $N$ [15]. The thermal radiation pressure in $D$ spatial dimensions is given by $P=e / D$ [16], yielding

$$
P=e / D=\frac{N \zeta(D+1) \Gamma\left(\frac{D+1}{2}\right) T^{D+1}}{\pi^{\frac{D+1}{2}} \hbar^{D}}
$$

Taking cognizance of Eqs. (11), (12), and (21), one can write Eq. (16) as

$$
W_{\mathrm{buoy}}=A \int_{l-b / 2}^{l+b / 2} \frac{N \zeta(D+1) \Gamma\left(\frac{D+1}{2}\right)(D-2) \hbar}{2^{D+2} \pi^{\frac{3 D+3}{2}} r_{H} l^{\prime} D} d l^{\prime} \text {. }
$$

Performing the integration in (22), one finds

$$
\begin{aligned}
& W_{\text {buoy }}=\frac{N A \zeta(D+1) \Gamma\left(\frac{D+1}{2}\right)(D-2) \hbar}{2^{D+2} \pi^{\frac{3 D+3}{2}}(D-1) r_{H}} \\
& \times\left[\left(l-\frac{b}{2}\right)^{-D+1}-\left(l+\frac{b}{2}\right)^{-D+1}\right]
\end{aligned}
$$

for the energy contribution due to the work done to overcome the quantum buoyancy. Finally, putting together Eqs. (13) and (23), one finds

$$
\begin{aligned}
& \mathcal{E}(l)=\frac{D-2}{2 r_{H}} \mu l+\frac{N A \zeta(D+1) \Gamma\left(\frac{D+1}{2}\right)(D-2) \hbar}{2^{D+2} \pi^{\frac{3 D+3}{2}}(D-1) r_{H}} \\
& \times\left[\left(l-\frac{b}{2}\right)^{-D+1}-\left(l+\frac{b}{2}\right)^{-D+1}\right]
\end{aligned}
$$

for the total energy of a body suspended at a proper distance $l$ above the horizon.

\section{THE NEUTRAL POINT}

The most challenging test of the GSL is obtained by dropping the object from the neutral (floating) point 
where $\mathcal{E}(l)$ reaches its minimum $[9-11]$. Setting $d \mathcal{E} / d l=$ 0 in (24), one obtains the condition determining the proper distance $l_{0}$ of the floating point from the horizon:

$$
\left(l_{0}-\frac{b}{2}\right)^{-D}-\left(l_{0}+\frac{b}{2}\right)^{-D}=\frac{2^{D+1} \pi^{\frac{3 D+3}{2}} \mu}{N A \zeta(D+1) \Gamma\left(\frac{D+1}{2}\right) \hbar} .
$$

Substituting (25) back into (24), one finds

$$
\begin{aligned}
& \mathcal{E}_{\min }=\frac{D-2}{2 r_{H}} \mu \\
& \times\left[l_{0}+\frac{1}{D-1} \frac{\left(l_{0}-\frac{b}{2}\right)^{-D+1}-\left(l_{0}+\frac{b}{2}\right)^{-D+1}}{\left(l_{0}-\frac{b}{2}\right)^{-D}-\left(l_{0}+\frac{b}{2}\right)^{-D}}\right]
\end{aligned}
$$

for the energy of the body at the floating point.

The universal bound on entropy, Eq. (2), can be deduced from the GSL only when the optimal drop point is close to the horizon, $l_{0}-b / 2 \ll b$. It is only in such situations that the contribution of quantum buoyancy to the energy of the body [the second term in Eq. [26)] is small.

Under which conditions does the neutral point lie in the near-horizon region? Substituting $l_{0}=b / 2+\epsilon b$ with $\epsilon \ll 1$ into Eqs. (25)-(26), one finds

$$
\mathcal{E}_{\min }=\frac{D-2}{4 r_{H}} \mu b\left[1+\frac{2 D}{D-1} \epsilon+O\left(\epsilon^{2}\right)\right],
$$

with

$$
\epsilon=\left[\frac{\hbar}{\mu b} \times \frac{N \zeta(D+1) \Gamma\left(\frac{D+1}{2}\right)}{2^{D+1} \pi^{\frac{3 D+3}{2}}}\right]^{1 / D} .
$$

Here we have used the relation $\left(l_{0}+b / 2\right)^{-D} \ll\left(l_{0}-\right.$ $b / 2)^{-D}$, which is valid in the near-horizon region, $l_{0}-$ $b / 2 \ll b$.

From Eq. (28) one learns that the neutral point lies in the near-horizon region (that is, $\epsilon \ll 1$ ) only if the size of the box is large enough such that

$$
\frac{b}{b_{C}}=\frac{N \zeta(D+1) \Gamma\left(\frac{D+1}{2}\right)}{2^{D+1} \pi^{\frac{3 D+3}{2}} \epsilon^{D}},
$$

where $b_{C} \equiv \hbar / \mu$ is the Compton length of the box. Note that the RHS of Eq. (29) increases very rapidly with the number $D$ of spatial dimensions: RHS $N\left(D / 8 e \pi^{3} \epsilon^{2}\right)^{D / 2} \gg 1$ for $D \gg 1$. This implies that for $D \gg 1$, the neutral point can lie in the near-horizon region only if the box is very large compared to its Compton length. As we shall now show, this severely restricts our ability to deduce the entropy bound (2) directly from the GSL in $(D+1)$-dimensional spacetimes with $D \gg 1$.

\section{THE ENTROPY BOUND}

The assimilation of the body by the black hole results in a change $\Delta \mathcal{M}=\mathcal{E}_{\min }$ in the mass of the black hole.
Using the relation $A_{H}=A_{D-1} r_{H}^{D-1}$ for the black hole's surface area together with Eqs. (6) and (27), one finds

$$
\left(\Delta A_{H}\right)_{\min }=4 \pi \mu b\left(1+\frac{2 D}{D-1} \epsilon\right)
$$

for the corresponding change in the surface area of the black hole. [Note that terms of order $(\Delta \mathcal{M} / \mathcal{M})^{2}$ are negligible for $b \ll r_{H}$.] Using the entropy-area relation for black holes, $S_{B H}=A_{H} / 4 \hbar$, one finds

$$
\left(\Delta S_{B H}\right)_{\min }=\frac{\pi \mu b}{\hbar}\left(1+\frac{2 D}{D-1} \epsilon\right)
$$

for the corresponding increase in the black hole's entropy.

Assuming the validity of the GSL [that is, $(\Delta S)_{\text {tot }} \equiv$ $\left.\left(\Delta S_{B H}\right)_{\min }-S \geq 0\right]$, one may deduce an upper bound on the entropy $S$ of a $(D+1)$-dimensional physical system of proper energy $E$ and proper length $b$ :

$$
S \leq \frac{\pi b E}{\hbar}\left(1+\frac{2 D}{D-1} \epsilon\right),
$$

where $\epsilon$ is determined in (28).

We see that the second term in (32), whose origins are in the quantum buoyancy, limits our ability to deduce the universal entropy bound in its canonical form (2). So, suppose one is willing to tolerate a small deviation from the canonical form (2) when deriving the entropy bound from the GSL. For example, assume that one wants to deduce from the present gedanken experiment an entropy bound of the form:

$$
S \leq(1+\delta) \times \frac{\pi b E}{\hbar},
$$

with $\delta \ll 1$. This amounts to the requirement that the magnitude of the work done to overcome the quantum buoyancy, Eq. (23), is limited by $\delta$ times the magnitude of the energy associated with the body's mass, Eq. (13). From (32) one finds that $\epsilon$ should take the small value $\epsilon=\delta(D-1) / 2 D$. From Eq. (28) with $\epsilon=\delta(D-1) / 2 D$ we learn that the entropy bound (33), which is weaker than the canonical bound (2) by the factor $1+\delta$, can be deduced from the GSL only for systems whose size is large enough such that

$$
b \geq \frac{\hbar}{\mu} \times F(D ; \delta),
$$

where

$$
F(D ; \delta) \equiv \frac{N \zeta(D+1) \Gamma\left(\frac{D+1}{2}\right) D^{D}}{2 \pi^{\frac{3 D+3}{2}}(D-1)^{D} \delta^{D}} ; \delta \ll 1 .
$$

As an example, let us take $\delta=10^{-1}$ in (33). This amounts to an entropy bound which is $10 \%$ weaker than the canonical form (2). For the familiar case of three spatial dimensions, one then finds a relatively small factor of $F(D=3) \approx 10^{1}$, which amounts to the restriction $b \gtrsim 10^{1} b_{C}$ on the size of the body. This implies 
that in three spatial dimensions, the entropy bound (33) with $\delta=10^{-1}$ can be derived from the GSL even for very small physical systems like atomic nuclei. However, one soon realizes that the function $F(D)$ in (35) increases very rapidly with the number of spatial dimensions: $F \approx 10^{5}, 10^{40}$, and $10^{91}$ for $D=10,50$, and 100 , respectively. This implies that the entropy bound (33) with $\delta=10^{-1}$ can be deduced from the GSL only for systems whose size is bounded from below according to: $b \gtrsim 10^{5} b_{C}, 10^{40} b_{C}$, and $10^{91} b_{C}$, respectively.

Had we taken $\delta=10^{-2}$ in (33), we would have found that an entropy bound which is $1 \%$ weaker than the canonical form (2) can be deduced from the GSL only for systems whose size is large enough such that $b \gtrsim$ $10^{4} b_{C}, 10^{15} b_{C}, 10^{90} b_{C}$, and $10^{191} b_{C}$ for $D=3,10,50$, and 100 , respectively.

\section{FLUID VS. WAVE PICTURE}

Are there any relevant effects which might change our conclusions? It was pointed out in [11] that the original derivation of the buoyant force from a fluid picture [9, 10] is valid if the characteristic wavelength $\bar{\lambda}$ in the thermal acceleration radiation is smaller than the box size $b$. At a fundamental level the buoyant force is caused by the momentum jolts the box receives as successive waves scatter off it [1]]. Long waves with $\lambda \gg b$ have difficulty matching specified boundary conditions on the surface of the box. Thus, they tend to scatter poorly and convey little momentum to the box [11]. One indeed finds that the wave scattering force is much weaker than the fluid force [11].

Note that the thermal distribution $\omega^{D} /\left(e^{\hbar \omega / T} \mp 1\right)$ of the acceleration radiation in Eq. (17) peaks at the characteristic frequency

$$
\bar{\omega}=\frac{D T}{\hbar}\left[1 \mp e^{-D}+O\left(e^{-2 D}\right)\right] .
$$

Taking cognizance of Eqs. (12) and (36), we find that the characteristic wavelength $\bar{\lambda}$ of the acceleration radiation at a proper distance $l$ above the horizon is

$$
\bar{\lambda} \simeq \frac{(2 \pi)^{2} l}{D}
$$

Thus, one realizes that the fluid picture of the acceleration radiation which was used in [9 11] (and which requires $\bar{\lambda} \lesssim b$ ) is valid in the region

$$
l \lesssim l(D)_{\text {fluid }} \equiv \frac{D}{(2 \pi)^{2}} b .
$$

From Eq. (38) one learns that the fluid region actually extends higher and higher above the black hole as the number $D$ of spatial dimensions becomes large. This implies, in particular, that at the near-horizon floating point, the larger is the value of $D$, the larger is the part of the box which is immersed in the fluid region. Thus, the larger is the value of $D$, the better is the fluid description. In fact, from (38) we learn that for $D \gtrsim(2 \pi)^{2}$ the entire box is already inside the fluid regime if the floating point is in the near-horizon region, $l_{0}-b / 2 \ll b$. Thus, for $D \gtrsim 40$ the use of the fluid picture in the near-horizon floating point is exact.

For $3 \leq D \lesssim 40$ and assuming that the floating point is in the near-horizon region, part of the body (the lower part) is in the fluid region (38) while part of it is in the (long) wave scattering regime. For $D$ values in this range, it is therefore more appropriate to perform the integration in (22) in the range $\left[l_{0}-b / 2, l(D)_{\text {fluid }}\right]$, where the fluid picture is valid:

$$
W_{\text {buoy }}=A \int_{l_{0}-b / 2}^{l(D)_{\text {fluid }}} \frac{N \zeta(D+1) \Gamma\left(\frac{D+1}{2}\right)(D-2) \hbar}{2^{D+2} \pi^{\frac{3 D+3}{2}} r_{H} l^{\prime} D} d l^{\prime} .
$$

This work should be corrected for the contribution from the rest of the box which is in the wave scattering regime. However, we know that the wave scattering force is much weaker than the fluid force [11, 17]. Hence, the integral in the range $\left[l_{0}-b / 2, l(D)_{\text {fluid }}\right]$ must give a close approximation to the true work. Indeed, this was verified by direct numerical computations in [11]. The physical reason for this success of the fluid picture (provided the floating point is in the near-horizon region, $\left.l_{0}-b / 2 \ll b\right)$ lies in the observation that the pressure drops precipitously as $l$ grows, $P \chi \propto l^{-D}$. This implies that the main contribution to the integrals in (22) and (39) comes from the lower part of the box, which is very close to the horizon well inside the fluid regime.

Performing the integration in (39), one finds that for $3 \leq D \lesssim 40$ the expression (26) for the energy of the body should be replaced by

$$
\begin{aligned}
& \mathcal{E}_{\min }=\frac{D-2}{2 r_{H}} \mu \\
& \times\left[l_{0}+\frac{b}{D-1} \frac{\epsilon^{-D+1}-\left(D / 4 \pi^{2}\right)^{-D+1}}{\epsilon^{-D}-\left(D / 4 \pi^{2}\right)^{-D}}\right] .
\end{aligned}
$$

Note that for all $D$ values, $\left(D / 4 \pi^{2}\right)^{-D} \ll \epsilon^{-D}$ in the near horizon limit, $\epsilon \ll 1$ (as explained above, this reflects the fact that the pressure drops precipitously as $l$ grows). For example, assume $\epsilon=(D-1) / 20 D$ which amounts to $\delta=10^{-1}$ in the bound (33). One then finds $\left(D / 4 \pi^{2}\right)^{-D} / \epsilon^{-D} \simeq 0.08,10^{-7}$, and $10^{-53}$ for $D=3,10$, and 40, respectively. Thus, one realizes that Eq. (40) reduces to (27) in the near-horizon limit for all $D$ values.

\section{SUMMARY}

We have considered a gedanken experiment in which a box full of entropy is lowered towards and then dropped into a $(D+1)$-dimensional black hole in equilibrium with thermal radiation. The effects of quantum buoyancy are parameterized by the function $F(D ; \delta)$ defined in Eqs. (34)-(35). We have seen that an entropy bound of the 
form (33) can be deduced from the GSL only if the system is large enough such that its size is larger than its Compton length by the factor $F(D ; \delta)$, see Eqs. (34)(35).

In the familiar case of three spatial dimensions, the factor $F$ is relatively small for $\delta=O\left(10^{-1}\right)$. This allows one to deduce an entropy bound which is $\sim 10 \%$ weaker than the canonical form (2) directly from the GSL for all macroscopic and mesoscopic objects (down to the scale of atomic nuclei). However, the function $F(D ; \delta)$ increases very rapidly with the number of spatial dimension: $F \sim$ $N\left(D / 2 \pi^{3} \delta^{2}\right)^{D / 2} \gg 1$ for $D \gg 1$. As a consequence, one finds that quantum buoyancy severely restricts our ability to deduce the universal entropy bound from the GSL in higher-dimensional spacetimes with $D \gg 1$.

In particular, for physical systems whose size lies in the range $b / b_{C}<F(D ; \delta)$, the entropy bound (2) cannot be derived directly from the GSL, not even in its weaker form (33). In other words, for such systems the entropy bound (2) does not serve as a necessary condition for the validity of the GSL. (Note, however, that the entropy bound (2) is always a sufficient condition for operation of the GSL in this type of gedanken experiments.) Finally, we note that our findings leave open the intriguing possibility of violating the universal entropy bound (2) in the range $b / b_{C} \leq F(D)$, without violating the generalized second law of thermodynamics.

\section{ACKNOWLEDGMENTS}

This research is supported by the Meltzer Science Foundation. I thank Yael Oren and Arbel M. Ongo for helpful discussions. I thank Jacob D. Bekenstein for helpful correspondence.
[1] D. Christodoulou, Phys. Rev. Lett. 25, 1596 (1970).

[2] S. W. Hawking, Phys. Rev. Lett. 26, 1344 (1971); S. W. Hawking, Commun. Math. Phys. 25, 152 (1972).

[3] J. M. Bardeen, B. Carter and S. W. Hawking, Commun. Math. Phys. 31, 161 (1973).

[4] J. D. Bekenstein, Lett. Nuov. Cim. 4, 737 (1972); J. D. Bekenstein, Phys. Rev. D 7, 2333 (1973); J. D. Bekenstein Phys. Rev. D 9, 3292 (1974).

[5] J. D. Bekenstein, Phys. Rev. D 23, 287 (1981).

[6] S. Hod, Phys. Rev. D 61, 024018 (2000) arXiv:gr-qc/9901035.

[7] J. D. Bekenstein and A. E. Mayo, Phys. Rev. D 61, 024022 (2000).

[8] S. Hod, Phys. Rev. D 61, 024023 (2000) arXiv:gr-qc/9903011.

[9] W. G. Unruh, Phys. Rev. D 14, 870 (1976); W. G. Unruh and R. M. Wald, Phys. Rev. D 25, 942 (1982); W. G.
Unruh and R. M. Wald, Phys. Rev. D 27, 2271 (1983).

[10] J. D. Bekenstein, Phys. Rev. D 49, 1912 (1994).

[11] J. D. Bekenstein, Phys. Rev. D 60, 124010 (1999).

[12] F. R. Tangherlini, Nuova Cimento 27, 636 (1963).

[13] G. Kunstatter, Phys. Rev. Lett. 90, 161301 (2003).

[14] R. Bousso, JHEP 0104, 035 (2001).

[15] V. Cardoso, M. Cavaglia, and L. Gualtieri, JHEP 0602, 021 (2006); H. Alnes, K. Olaussen, F. Ravndal, and I.K. Wehus, J. Phys. A 40, F315 (2007).

[16] H. Alnes, F. Ravndal, and I.K. Wehus, J. Phys. A 40, 14309 (2007).

[17] As explained above, long waves have difficulty matching specified boundary conditions on the object's surface. As a consequence, they tend to scatter poorly and convey little momentum to the object [11]. 